\title{
EDIFİCATE
}

I Congreso de Escuelas de Edificación y Arquitectura Técnica de España València, 4 y 5 de noviembre de 2021

Escuela Técnica Superior de Ingeniería de Edificación

Universitat Politècnica de València

Doi: https://doi.org/10.4995/EDIFICATE2021.2021.13599

\section{Propuesta de modificación de las Competencias de la asignatura Construcción VI de la Escuela Técnica Superior de Ingeniería de Edificación de la Universitat Politècnica de València}

\section{Proposal to modify the Competences of the Construction VI course of the Higher Technical School of Building Engineering of the Polytechnic University of Valencia}

\begin{abstract}
Jaime Llinares Millán ${ }^{a}$, José Miguel Molines Cano ${ }^{b}$ y José María Bravo Plana-Salac
aUniversitat Politècnica de València, Departamento de Construcciones Arquitectónicas, jllinares@csa.upv.es, bUniversitat Politècnica de València, Departamento de Mecánica de los Medios Continuos y Teoríad e Estructuras, jomoca1@upv.es y 'Universitat Politècnica de València, Departamento de Física Aplicada, jpbrapla@fis.upv.es.
\end{abstract}

\begin{abstract}
In the following work a modification of the competences of the Construction VI subject is presented. The modification focuses on two fundamental aspects; redefine the specific competences of the subject; and divide these competencies into learning outcomes that make sense with the evolution of student learning.
\end{abstract}

Keywords: Construction VI; Building pathology; Transversal competences; Learning outcomes

\section{Resumen}

En el siguiente trabajo se presenta una modificación de las competencias de la asignatura Construcción VI. La modificación se centra en dos aspectos fundamentales; redefinir las competencias específicas de la asignatura; $y$ dividir estas competencias en resultados de aprendizaje que tengan sentido con la evolución del aprendizaje del alumno.

Palabras clave: Construcción VI; Patología de la edificación; Competencias transversales; Resultados del aprendizaje 


\section{Descripción general de la asignatura}

\subsection{Ubicación de la asignatura dentro de la titulación}

Entre las asignaturas que el alumno debe cursar para obtener el título de Grado de Arquitectura Técnica (G.A.T.) se encuentra la asignatura obligatoria "Construcción VI", con 4,5 créditos ECTs, adscrita al Departamento de Construcciones Arquitectónicas (ANECA, 2005).

Según el Plan de Estudios del G.A.T., la asignatura se ubica en el primer semestre del cuarto curso, lo que implica que los conocimientos de construcción que tienen los estudiantes cuando empiezan a cursarla son los adquiridos en los tres cursos anteriores en las asignaturas de Materiales de Construcción I, II y III y las de Construcción I, II-III y IV-V.

En relación con este aspecto, la asignatura Construcción $\mathrm{VI}$, pese a continuar la línea docente de las asignaturas de Construcción anteriores (I, II-III y IV-V), es la única cuya temática puede entenderse como "complementaria" o como "módulo adicional" ya que no cierra ningún proceso anterior, sino que prácticamente supone una competencia por si misma (a falta de ciertos resultados de aprendizaje correspondientes a la asignatura "Construcciones históricas") (ANECA, 2005).

No obstante, pese a ser un módulo diferenciado hay que señalar que para la comprensión de los contenidos de "Construcción VI", son necesarios, además de los conocimientos de construcción impartidos en las asignaturas de cursos precedentes, unos conocimientos básicos de Química, Física de los Materiales de Construcción, Estructuras y Seguridad y Salud. Estas materias son objeto de estudio en asignaturas anteriores del G.I.E. por lo que, en tanto no se modifique su ubicación, no habrá que incluirlos dentro del programa de "Construcción VI". También hay que señalar que la formación constructiva obligatoria del Grado en Arquitectura Técnica, finaliza con esta asignatura, pudiéndose completar con el área de intensificación "Intervención en construcciones históricas".

\subsection{Contenido y explicación general de la asignatura}

Tal como se puede observar en la figura 1, la disciplina de Construcción es uno de los pilares fundamentales de la titulación de Grado en Arquitectura Técnica, siendo la única de todas ellas que se encuentra presente en todos los semestres.

En la asignatura "Construcción I" se estudia el vocabulario y las soluciones básicas de la construcción. En la asignatura "Construcción II" el alumno aprende cómo funcionan estas soluciones al detalle, razonando para qué sirve cada uno de los componentes que las forman. La evolución lógica viene con la asignatura "Construcción III", en la cual, tras haber aprendido cómo funcionan y que misión tiene cada uno de los componentes que conformar una solución constructiva, el alumno tiene que aprender a formular soluciones constructivas nuevas, diseñadas o razonadas para una situación o problemática específica. 


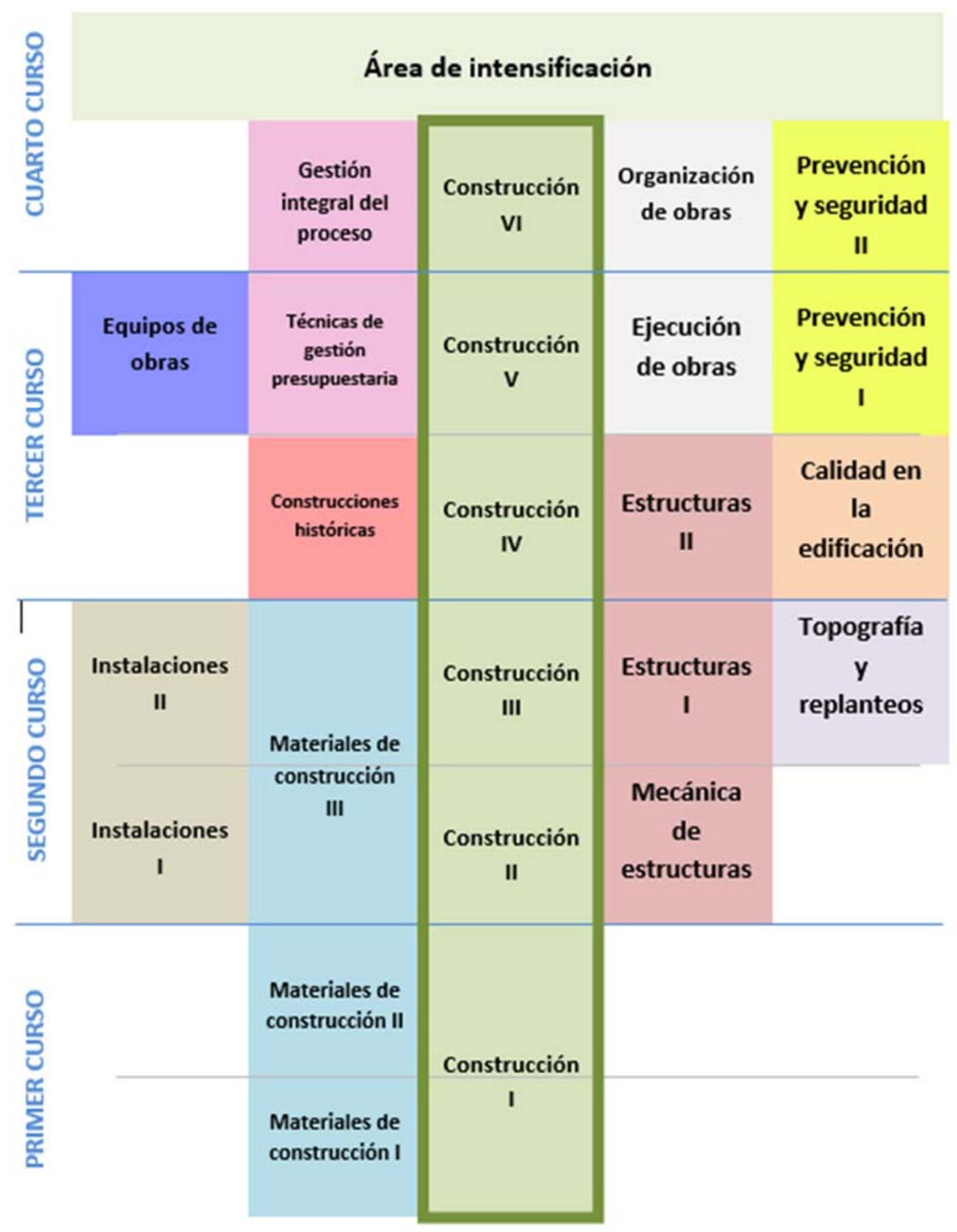

Fig. 1 Esquema de la distribución temporal de las asignaturas de Construcción, dentro del Área de intensificación en la titulación de Grado de Arquitectura Técnica en la ETSIE de la UPV.

Fuente: El autor 
Llegados a este punto, cuando el alumno ya comprende las diferentes soluciones y procesos constructivos ( $\mathrm{y}$ de forma detallada cómo funciona cada componente), en la asignatura "Construcción IV" se integran estos procesos dentro del funcionamiento global de la obra. Continuando con la visión de la obra como un proceso único compuesto por pequeños procesos relacionados entre sí, la asignatura "Construcción V" completa esta primera fase de la disciplina de la construcción introduciendo los medios secundarios y auxiliares de la obra (remates, grúas, seguridad y salud, etc...).

La asignatura "Construcción VI" tiene como objetivo principal abordar la temática referente al mantenimiento y restauración de los elementos construidos. En primer lugar se estudian las diferentes filosofías y sensibilidades en el campo de la intervención de elementos construidos, así como las técnicas de diagnosis más empleadas actualmente. En una segunda fase se estudian las diferentes lesiones que pueden encontrarse en los distintos elementos que componen un edificio (siempre que sea posible se intentarán agrupar por temática similar en cuanto a zonificación o elemento dañado). Finalmente, la asignatura se cierra con la redacción del libro de mantenimiento del edificio (ANECA, 2008).

Tal como puede observarse, pese a que es necesario tener los conocimientos y competencias adquiridas en el resto de las asignaturas de la disciplina (Construcción I, II, III, IV y V), la asignatura no sigue la linealidad que siguen el resto, siendo en sí misma un módulo independiente. Esta idea de independencia se acusa al comparar la asignatura y buscar sus homónimas en los planes de estudio de las diferentes escuelas técnicas de Ingeniería de Edificación de España.

En algunas escuelas, como en la Politécnica de Barcelona, se mantiene la misma nomenclatura, Construcción VI (primer cuatrimestre de cuarto curso), con un temario prácticamente idéntico. En otros casos se refuerza el concepto de mantenimiento del edificio, como es el caso de la Universidad Politécnica de Madrid, en la que dividen los contenidos en dos asignaturas distintas. Por una lado, la asignatura "Patología y Rehabilitación" (primer cuatrimestre de cuarto curso) y por otro, la asignatura "Mantenimiento" (segundo cuatrimestre de cuarto curso). Hay que señalar que la suma de los créditos de ambas asignaturas es muy similar a los que tiene "Construcción VI".

En otras escuelas se ha decidido eliminar el módulo de Mantenimiento para poder ahondar más en el módulo de Diagnosis y Patología. Este es el caso de la Universidad de Sevilla y su asignatura "Restauración y Patología de la Edificación (primer cuatrimestre de cuarto curso). Finalmente, en algunas escuelas se ha optado por darle un enfoque más humanístico, unificando la disciplina de la intervención con la historia de la arquitectura local. Un ejemplo de este último grupo es la Universidad de la Coruña con la asignatura "Patrimonio Arquitectónico Gallego", centrada, sobre todo, en las intervenciones realizadas en los monumentos más significativos de Galicia. 
Propuesta de modificación docente de la asignatura Construcción VI de la Escuela Técnica Superior de Ingeniería de Edificación de la Universitat Politècnica de València - Proposal for teaching modification of the Construction VI subject of the Higher Technical School of Building Engineering of the Polytechnic University of Valencia

\section{Competencias}

\subsection{Genéricas}

En el plan de estudios actual, se establecen las siguientes competencias genéricas para la asignatura:

- Poseer y comprender conocimientos en un área de estudio que parte de la base de la educación secundaria general, y se suelen impartir a un nivel, que si bien se apoya en libros de texto avanzados, incluye también algunos aspectos que implican conocimientos procedentes de la vanguardia de su campo de estudio.

- Saber aplicar sus conocimientos a su trabajo o vocación de una forma profesional y poseer las competencias que suelen demostrarse por medio de la elaboración y defensa de argumentos y resolución de problemas dentro del área de estudio.

- Capacidad de reunir e interpretar datos relevantes (normalmente dentro de su área de estudio) para emitir juicios que incluyan una reflexión sobre temas relevantes de índole social, científica o ética.

- Capacidad de transmitir información oral y escrita en la lengua nativa: ideas, problemas y soluciones a un público tanto especializado como no especializado.

- Poseer habilidades de aprendizaje necesarias para emprender estudios posteriores con un alto grado de autonomía.

- Conducta ética en ingeniería. Capacidad crítica y autocrítica. Conciencia de la necesidad de calidad y alto nivel profesional y de su aplicación al continuo perfeccionamiento.

Sin embargo, según las recomendaciones establecidas en el III Congreso Internacional sobre competencias docentes (Berna, 2012), es conveniente reformular las competencias de las asignaturas para que no superen las seis competencias genéricas, y una específica. Del mismo modo, se aconseja establecer títulos más globales para nombrar las competencias genéricas. Por ello, revisando las competencias actuales y los objetivos de la asignatura se decide reformularlas siendo el resultado el siguiente (Ausubel, 1990):

- Sostenibilidad y compromiso social. Tener en cuenta las dimensiones social, económica y ambiental al aplicar soluciones y llevar a cabo proyectos coherentes con el desarrollo humano y la sostenibilidad.

- Comunicación eficaz oral y escrita. Comunicarse de manera clara y eficiente en presentaciones orales y escritas adaptadas al tipo de público y a los objetivos de la comunicación utilizando las estrategias y los medios adecuados.

- Trabajo en equipo. Dirigir y dinamizar grupos de trabajo, resolviendo posibles conflictos, valorando el trabajo hecho con las otras personas y evaluando la efectividad del equipo así como la presentación de los resultados generados. 
- Aprendizaje autónomo. Aplicar los conocimientos alcanzados en la realización de una tarea en función de la pertinencia y la importancia, decidiendo la manera de llevarla a cabo y el tiempo que es necesario dedicarle y seleccionando las fuentes de información más adecuadas.

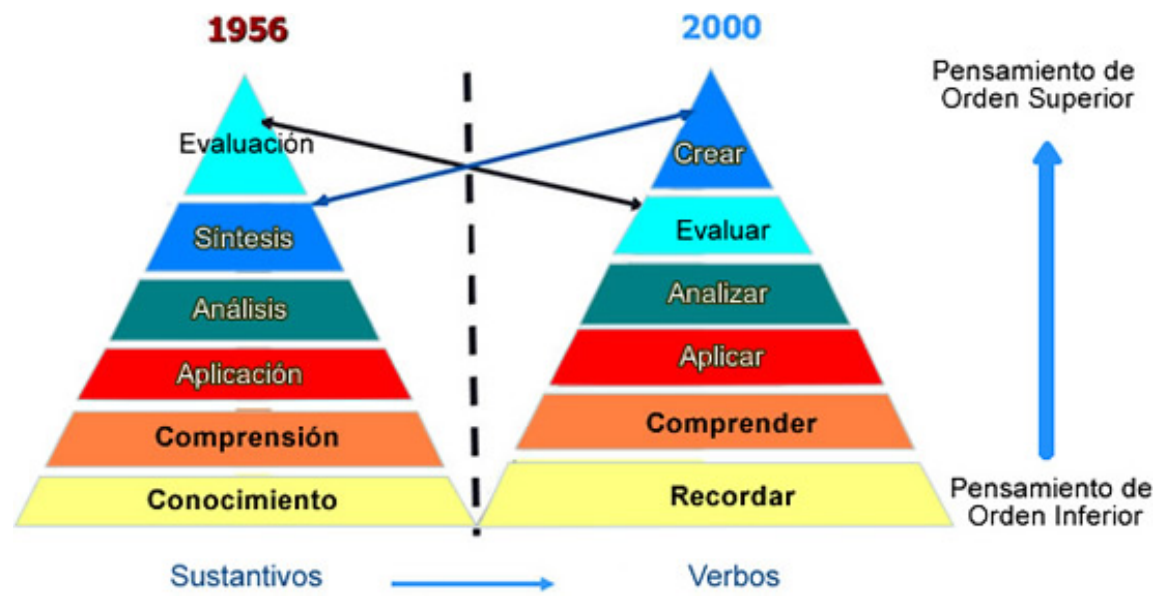

Fig. 2 Taxonomía de Bloom sobre los niveles de las habilidades del pensamiento. Se compara la taxonomía oficial de 1956 basada en sustantivos y su actualización del año 2000 basada en verbos. Fuente: Instituto de Ciencias de la Educación. UPV.

A la hora de reformularlas se tiene en cuenta el nivel de resultados de aprendizaje alcanzados en anteriores asignaturas, por lo que, aplicando la taxonomía de Bloom, todas las competencias se encuentran en el estadio 3 o 4 (Ausubel, 1990).

\subsection{Específicas}

En el bloque de las competencias específicas ocurre exactamente los mismo que con las competencias genéricas, que no se corresponden de forma coherente o precisa con las competencias propias de la asignatura.

Siguiendo el mismo esquema que en el apartado anterior, se listan las competencias específicas establecidas para la asignatura por el plan de estudios actual:

- Conocimiento de los elementos, sistemas y tipologías constructivas, tradicionales y prefabricados empleados en la edificación y sus variedades.

- Conocimiento de la evolución histórica de las técnicas, elementos y procedimientos constructivos y los sistemas estructurales que han dado origen a las formas estilísticas.

- Aptitud para identificar los elementos, sistemas y tipologías constructivas, definir su función, idoneidad relativa y compatibilidad en el proceso constructivo. Aptitud para plantear y resolver detalles constructivos. 
Propuesta de modificación docente de la asignatura Construcción VI de la Escuela Técnica Superior de Ingeniería de Edificación de la Universitat Politècnica de València - Proposal for teaching modification of the Construction VI subject of the Higher Technical School of Building Engineering of the Polytechnic University of Valencia

- Conocimiento de los procedimientos específicos de control de la ejecución material de obras de edificación.

- Capacidad para dictaminar sobre las causas y manifestaciones de las lesiones en los edificios, y proponer soluciones para evitar o subsanar su patología.

- Aptitud para evaluar e intervenir en la rehabilitación de edificios y en la conservación y restauración del patrimonio construido. Conocimiento del marco legislativo, normativa técnica y doctrina específica, de aplicación en el patrimonio construido.

- Capacidad para el análisis del ciclo de vida útil de los elementos y sistemas constructivos de los edificios.

- Capacidad para elaborar manuales y planes de mantenimiento de edificios y gestionar su implantación en el edificio.

- Conocimiento de la evaluación del impacto medioambiental de los procesos de edificación y demolición y de la sostenibilidad en la edificación.

- Conocimiento de los procedimientos y técnicas de evaluación de la eficiencia energética de los edificios. Aptitud para diseñar y materializar soluciones de acondicionamiento acústico, térmico y lumínico de los mismos.

- Capacidad para aplicar la normativa técnica al proceso de la edificación y generar documentos de especificación técnica que determinen los procedimientos y métodos constructivos de edificios.

- Conocimiento de los equipos, instalaciones provisionales y medios auxiliares empleados en las obras de edificación, sus características y normativa de aplicación. Capacidad para evaluar las necesidades y establecer criterios para la selección de los equipos, instalaciones provisionales y medios auxiliares.

Siguiendo las mismas pautas que en el apartado anterior, resulta necesario reformular las competencias, no sólo las referentes a la asignatura (pasaremos de 12 competencias específicas a 1, la cual la dividiremos a su vez en 5 resultados de aprendizaje). El resultado sería una única competencia específica principal, cuyos objetivos de aprendizaje prácticamente se vean abarcados en su totalidad por la asignatura (Fowler, 2002).

Para fijar la competencia específica principal, se buscan los dos elementos claves que definen la asignatura, el estudio del estado patológico, y el aprendizaje de métodos o técnicas para poder intervenir las lesiones diagnosticadas. En base a estos dos elementos, y aplicando el mismo proceso descriptivo basado en la taxonomía de Bloom se redacta una única competencia específica principal (que luego se dividirá en varios resultados de aprendizaje). (Elliot, 2000).

Competencia específica principal: Diagnosticar la patología de elementos edificados para su futura intervención. (Competencia adquirida en las asignaturas Construcción VI e Intervención en construcciones históricas).

El desglose en resultados de aprendizaje sería el siguiente: 
Tabla 1. Desglose de la competencia principal en sus respectivos resultados de aprendizaje, y su distribución dentro de las diferentes asignaturas que la componen. Fuente: El autor.

\begin{tabular}{l|c|c|}
\multicolumn{1}{|c|}{ Resultado de aprendizaje } & C. VI & I. en C.H. \\
\hline $\begin{array}{l}\text { Identificar las diferentes lesiones patentes en elementos construidos } \\
\text { Evaluar el daño/nivel de afección de cada lesión en elementos } \\
\text { construidos }\end{array}$ & $\checkmark$ & $\boldsymbol{X}$ \\
\hline $\begin{array}{l}\text { Realizar mapeados de lesiones aplicados sobre elementos } \\
\text { construidos }\end{array}$ & $\checkmark$ & $\boldsymbol{X}$ \\
\hline $\begin{array}{l}\text { Proponer posibles soluciones/intervenciones para las diferentes } \\
\text { lesiones }\end{array}$ & $\checkmark$ & $\boldsymbol{X}$ \\
\hline $\begin{array}{l}\text { Escoger y razonar entre las diferentes opciones de intervención } \\
\text { interpretando los datos obtenidos en estudios previos }\end{array}$ & $\boldsymbol{x}$ \\
\hline
\end{tabular}

\section{Indicadores de logro}

Para que los alumnos alcancen estas competencias, a continuación se exponen los objetivos específicos, o indicadores de logro, que han de superar los alumnos al cursar esta asignatura. Dichos indicadores servirán para evaluación el grado de conocimiento y habilidades adquiridos por los alumnos, así como la efectividad de la planificación docente (Elliot, 2000).

Tabla 2. Indicadores de logro definidos para la asignatura "Construcción Vl". Fuente: El autor.

\begin{tabular}{|l|l|l|}
\hline Código & \multicolumn{1}{|c|}{ Indicador de logro } & \multicolumn{1}{|c|}{ Recursos } \\
\hline IL 1 & $\begin{array}{l}\text { Introducir al alumno en las diferentes } \\
\text { filosofías de intervención existentes. }\end{array}$ & Clases teóricas \\
\hline IL 2 & $\begin{array}{l}\text { Conocimiento sobre las diferentes técnicas de } \\
\text { diagnosis existentes en la actualidad. Ensayos } \\
\text { no destructivos y semidestructivos }\end{array}$ & $\begin{array}{c}\text { Clases teóricas, sesiones en } \\
\text { el laboratorio, seminarios } \\
\text { específicos. }\end{array}$ \\
\hline
\end{tabular}


Propuesta de modificación docente de la asignatura Construcción VI de la Escuela Técnica Superior de Ingeniería de Edificación de la Universitat Politècnica de València - Proposal for teaching modification of the Construction VI subject of the Higher Technical School of Building Engineering of the Polytechnic University of Valencia

\begin{tabular}{|c|c|c|}
\hline Código & Indicador de logro & Recursos \\
\hline IL 3 & $\begin{array}{l}\text { Comprensión de la patología (conjunto de } \\
\text { lesiones) existente por causas estructurales }\end{array}$ & $\begin{array}{l}\text { Clases teóricas, prácticas de } \\
\text { campo, visitas temáticas }\end{array}$ \\
\hline IL 4 & $\begin{array}{l}\text { Comprensión de la patología (conjunto de } \\
\text { lesiones) existente por causas no estructurales }\end{array}$ & $\begin{array}{l}\text { Clases teóricas, prácticas de } \\
\text { campo, visitas temáticas }\end{array}$ \\
\hline IL 5 & $\begin{array}{l}\text { Conocimiento del alcance superficial de cada } \\
\text { lesión. Elementos que intervienen en una } \\
\text { lesión }\end{array}$ & $\begin{array}{l}\text { Clases teóricas, seminarios } \\
\text { específicos impartidos por } \\
\text { expertos del sector }\end{array}$ \\
\hline IL 6 & $\begin{array}{l}\text { Prevención de futuros daños en elementos } \\
\text { cercanos a una lesión no dañados }\end{array}$ & $\begin{array}{l}\text { Clases teóricas, prácticas de } \\
\text { campo }\end{array}$ \\
\hline IL 7 & $\begin{array}{l}\text { Detectar el origen y las causas de las } \\
\text { diferentes lesiones de un edificio. }\end{array}$ & $\begin{array}{l}\text { Clases teóricas, estudio de } \\
\text { casos, trabajo en grupo }\end{array}$ \\
\hline IL 8 & $\begin{array}{l}\text { Relacionar las diferentes lesiones de un } \\
\text { edificio, identificando las causas y efectos } \\
\text { secundarios comunes. }\end{array}$ & $\begin{array}{l}\text { Clases teóricas, estudio de } \\
\text { casos, trabajo en grupo }\end{array}$ \\
\hline IL 9 & $\begin{array}{l}\text { Proponer diferentes soluciones y realizar el } \\
\text { pertinente proyecto de intervención }\end{array}$ & $\begin{array}{l}\text { Clases teóricas, estudio de } \\
\text { caso. }\end{array}$ \\
\hline IL 10 & $\begin{array}{l}\text { Redactar el libro de mantenimiento de un } \\
\text { edificio }\end{array}$ & Clases teóricas \\
\hline
\end{tabular}

\section{Conclusión. Relación entre las Competencias y los Indicadores de logro.}

A continuación, se expone la relación entre las competencias (las cuatro competencias genéricas y la competencia específica anteriormente descritas) de la asignatura "Construcción Vl", y los objetivos específicos o indicadores de logro (Fowler, 2002).

Hay que destacar el hecho de que no existe ningún indicador de logro que esté sujeto o restringido a una única competencia (genérica o específica), por lo que se cumplen los 
preceptos de compatibilidad y complementariedad de competencias fijados en el III Congreso Internacional sobre competencias docentes (Berna, 2012).

Tabla 3. Relación entre los objetivos específicos (indicadores de logro) y las competencias de "Construcción Vl". Fuente: El autor.

\begin{tabular}{|c|c|c|c|c|c|}
\hline & CG 1 & CG 2 & CG 3 & CG 4 & $C E$ \\
\hline IL 1 & $\checkmark$ & $x$ & $x$ & $x$ & $\checkmark$ \\
\hline IL 2 & $\checkmark$ & $\checkmark$ & $\checkmark$ & $x$ & $\checkmark$ \\
\hline IL 3 & $x$ & $\checkmark$ & $x$ & $x$ & $\checkmark$ \\
\hline IL 4 & $x$ & $x$ & $x$ & $\checkmark$ & $\checkmark$ \\
\hline IL 5 & $x$ & $x$ & $x$ & $\checkmark$ & $\checkmark$ \\
\hline IL 6 & $x$ & $x$ & $x$ & $\checkmark$ & $\checkmark$ \\
\hline IL 7 & $x$ & $\checkmark$ & $\checkmark$ & $x$ & $\checkmark$ \\
\hline$I L 8$ & $x$ & $\checkmark$ & $\checkmark$ & $x$ & $\checkmark$ \\
\hline IL 9 & $\checkmark$ & $\checkmark$ & $\checkmark$ & $x$ & $\checkmark$ \\
\hline IL 10 & $x$ & $\checkmark$ & $x$ & $x$ & $\checkmark$ \\
\hline
\end{tabular}

\section{Referencias}

ANECA. (2008). Programa academia: principios y orientaciones para la aplicación de los criterios de evaluación. Madrid: Agencia Nacional de Evaluación del Profesorado.

ANECA. (2005). Libro blanco. Título de grado en ingeniería de edificación. Madrid: Agencia Nacional de Evaluación de la Calidad y Acreditación.

AUSUBEL D.P. (1990). Significado y aprendizaje significativo. In: Ausubel D.P., Novak J.D., Hanesian H. (eds) Psicología educativa: un punto de vista cognoscitivo. Trillas, Mexico, pp 55-107.

ELLIOT J. (2000). El cambio educativo desde la investigación-acción (traducción de P. Manzano). Madrid : Morata.

FOWLER B. (2002). La taxonomía de Bloom y el pensamiento crítico. http://www.eduteka.org/profeinvitad.php3?ProflnvID=0014 (acceso 15.01.2010) 